\title{
Lipoperoxidation and vitamin C status in psoriasis
}

\author{
MirceaTampa ${ }^{1,2}$, llinca Nicolae ${ }^{2}$, Clara Matei ${ }^{1}$, Maria Isabela Sarbu ${ }^{1}$, Corina-Daniela \\ Ene $^{3}$, Simona-Roxana Georgescu ${ }^{1,2}$
}

\section{1-"Carol Davila" University of Medicine and Pharmacy \\ 2-Department Dermatology, "Victor Babes" Clinical Hospital of Tropical and Infectious \\ Diseases, Bucharest, Romania \\ 3-Nephrology Hospital “Dr. Carol Davila”, Bucharest, Romania}

Oxidative stress is caused by an imbalance between the production of pro-oxidants and the capacity of a biological system to rapidly detoxify the free radicals, remove altered molecules and repair the resulted prejudice. Oxidative stress is not an affliction, a symptom or a syndrome, but a biochemical process generated and maintained by exogenous and endogenous factors. In this paper we quantified the level of pro-oxidants and antioxidants in patients with psoriasis vulgaris.

The urinary levels of lipid peroxides (expressed through the concentration of thiobarbituric acid reactivesubstances-TBARs), creatinine and vitamin $C$ were determined in 90 patients (40 patients with active psoriasis and 50 patients with remitted psoriasis) and 50 controls.

The level of vitamin $\mathrm{C}(\mathrm{mg} / \mathrm{dL})$ was decreased in patients with psoriasis as compared to the controls (11.3 \pm 6.8 versus 17.3 $\pm 10.4, p=0.099$ ) (Figure 1). Different values were also obtained between patients with active and remitted psoriasis $(8.8 \pm 3.7$ versus $13.2 \pm 8.7, p=0.062)$. The level of lipid peroxides (ng TBARs/mg creatinine) presented considerable differences between patients with psoriasis and controls (14.7 \pm 6.2 compared to $7.5 \pm 3.1$, $\mathrm{p}=0.016$ ) (Figure 2) and between active and remitted psoriasis (18.3 \pm 8.2 compared to $11.5 \pm 3.2, p=0.031)$. A negative association between the concentration of vitamin $\mathrm{C}$ and the TBARs/ creatinine ratio was found through simple linear regression analysis in patients with active psoriasis $(r=-0.602, p=0.009)$ and remitted psoriasis $(r=-0.266 . p=0.047)$.
The results of the study show that the level of reactive oxygen species dramatically decreases and physiological antioxidant mechanisms are inefficient in patients with psoriasis vulgaris. These findings reconfirm that oxidative stress has a destructive and pathogenic role in psoriasis.

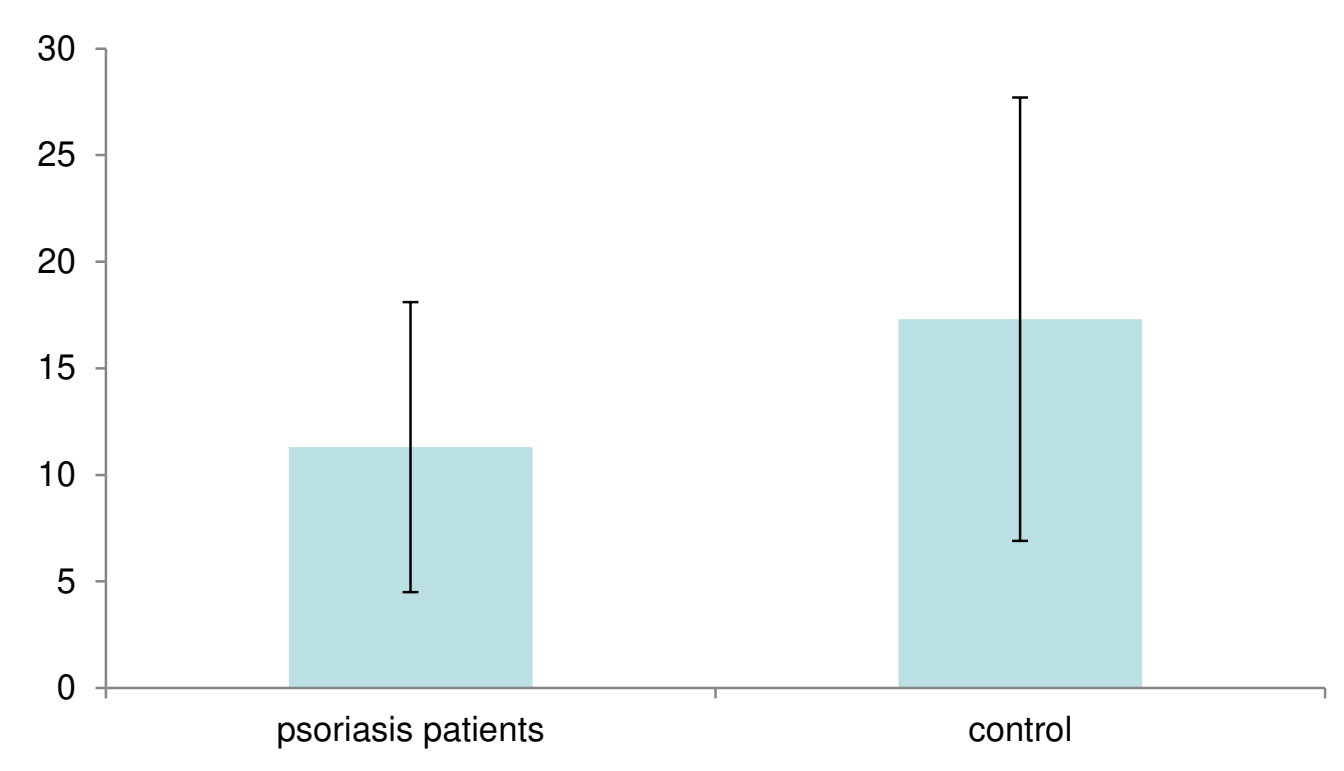

Figure 1. The level of vitamin $C$ in psoriasis patients versus controls

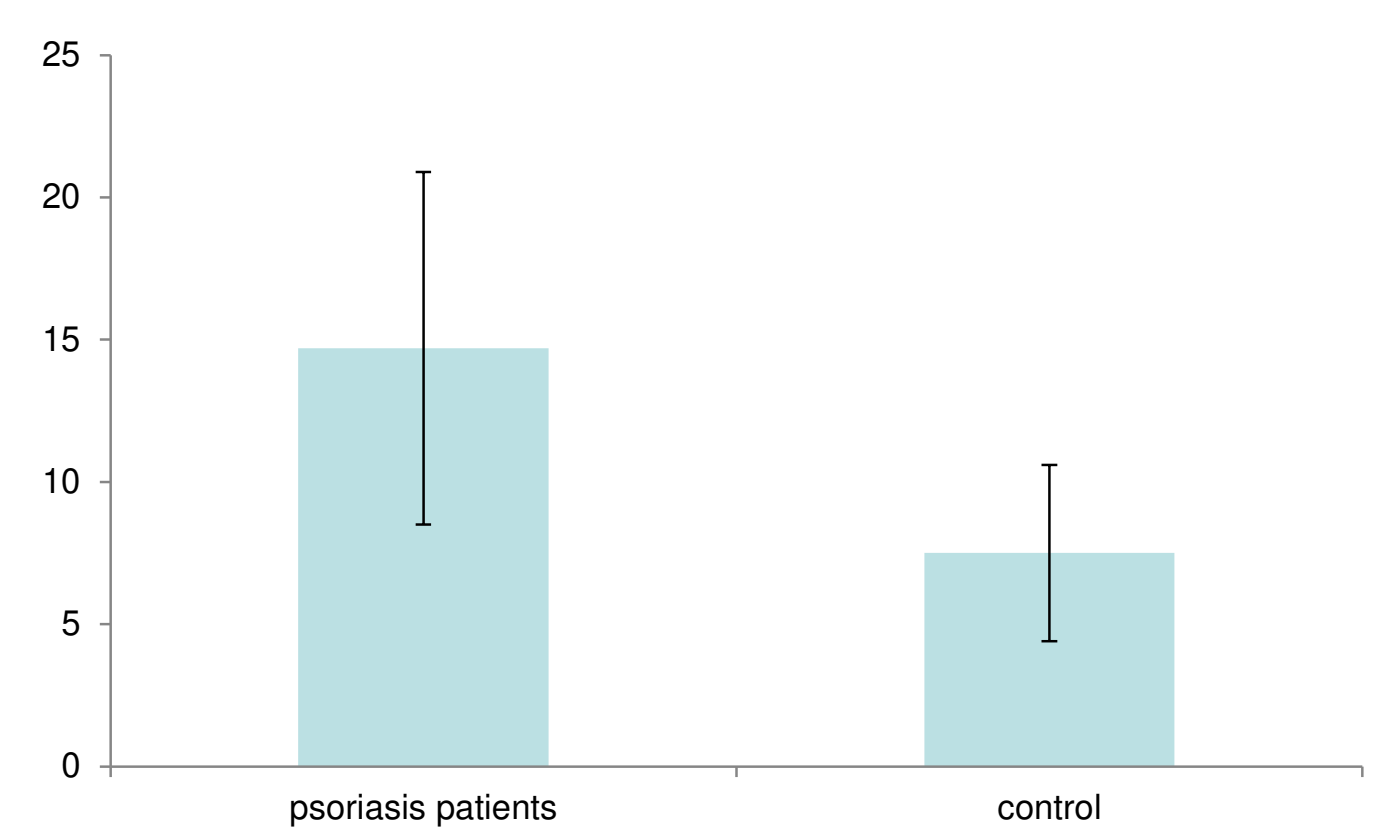

Figure 2. The level of lipid peroxides in psoriasis patients versus controls 